\title{
Exposición a televisión, uso de medios impresos y razonamiento verbal entre jóvenes universitarios
}

Dora Martínez Ramos

Universidad de Monterrey (UDEM)

BuEna PARTE de los estudios sobre efectos de televisión y niños en diversos paísesse han centrado primordialmente en alguno de los siguientes temas: agresión, consumismo, fomento de estereotipos e influencias culturales (Cullingford, 1984; Greenfield, 1984; Moody, 1980; Medina, 1986; Rebeil, 1986; Dorr,1990, Neuman, 1990).

Muchos de ellos han surgido frente a necesidades específicas, y han encontrado el respaldo de fundaciones y gobiernos, como sucedió en Estados Unidos en 1950 y 1971 (Véase Liebert y Sprafkin, 1988). La investigación sobre televisión y niños, sobre todo en países donde se dedica más presupuesto a ello, ha seguido cauces mayormente marcados por cuestiones de mercado, de opinión pública, o inclușo de moda intelectual (véase el boom actual de los estudios de género, en Inglaterra, o de mediaciones culturales en Latinoamérica).

Sin embargo, los resultados de estos estudios, particularmente los realizados desde una perspectiva experimental en busca de evidencias de relación causal entre la televisión y diferentes efectos, han sido en su mayoría contradictorios. La revisión de literatura que algunos autores han realizado en torno a estos temas concluye por lo general en que no existe suficiente evidencia empírica de que la televisión realmente incite a los menores a la agresión o a la adopción ciega de los patrones de comportamiento individuales o culturales que se les proponen (Surgeon General Report, 1971; Schramm, Lyle y Parker, 1961; Cullingford, 1984; Corona, 1986).

Los críticos más cautelosos sugieren que no se puede tampoco concluir que no existen tales efectos, y que lo que hace falta es desarrollar una metodología capaz de detectarlos (Fernández, 1986; Dorr,1990) Por otra parte, son menos abundantes 
los estudios que tratan de determinar los efectos de la televisión en áreas más específicas, relacionadas con procesos mentales, como la percepción, atención, reflexión, y en general, el impacto del medio en el desarrollo cognitivo y en el desarrollo de habilidades de comunicación lingüística particulares, como la lectura y la escritura (Orozco, 1985; Armstrong, 1990; Bryant, 1983). Es más fácil encontrar trabajos dedicados a explorar las potencialidades educativas del medio, aunque sus resultados no son tampoco muy optimistas (Minton, 1972; Alvarez, 1980; Charles, 1986; Chávez, 1985; Crovi, 1985; Orozco, 1986; Lesser, 1975; Cullingford, 1984). La mayoría de los estudios mexicanos revisados en el área se refieren a las potencialidades o a las fallas educativas de la televisión, así como a efectos en los procesos de socialización (Fernández, 1986; Sánchez, 1989) Otros pocos analizan, de manera más teórica que empírica, el posible impacto cognoscitivode la televisión, sin establecer claramente el mecanismo causal de este impacto (Orozco, 1985 y 1986).

\section{Televisión y lectoescritura}

La enseñanza de la lectura y la escritura ha tenido primacía y aparece en todos los currícula desde la escuela primaria hasta los primeros años de universidad. Sin embargo, las presiones tecnológicas de nuestra época, han provocado que se les dé más importancia a los conocimientos relativos a las ciencias exactas, haciendo de lado todo lo relacionado con las letras, por considerarlo improductivo, ocioso $o$ inútil.

Si se considera la cantidad de años que se dedican en la escuela a enseñarnos a escribir y leer parece sorprendente que los resultados sean tan lamentables.

Según estudios recientes realizados en México (Rodríguez,1990; González, 1986; Basave y Martínez, 1980) hay evidencias de que los estudiantes preparatorianos y universitarios mexicanos presentan grandes deficiencias en el manejo de la lengua, lo cual repercute en las habilidades generales de razonamiento verbal y en la calidad del aprendizaje que se lleva a cabo en la escuela.

En particular, el estudio de Rodríguez (1990) muestra que los estudiantes no están capacitados, en materia de lenguaje, para los estudios universitarios. Al no saber leer ni escribir de manera adecuada, los jóvenes están incapacitados para ejercitar su capacidad de análisis, y para expresar o formular juicios críticos. Esto, 
según la autora, refleja una problemática que tiene sus raíces en la educación básica, primaria y secundaria, y que aparece no sólo a nivel local, sino mundial.

Podríamos señalar al menos tres posibles fuentes de impacto sobre las habilidades verbales de los jóvenes: el medio familiar (Antinori, 1989), el sistema educativo y los métodos de enseñanza (Gutiérrez, 1986, Rodríguez, 1990) y el consumo de tecnologías de comunicación audiovisual - predominio de la imagen sobre la palabra - ( Van Peer, 1988; Neuman, 1988; Beentjes y Van der Voort, 1988; Neuman, 1991).

En este artículo nos centraremos en eltercer factor de influencia, la preeminencia de la imagen sobre lo impreso. Varios autores han observado que desde la aparición de la televisión en nuestras sociedades, parece que la actitud hacia lo impreso ha cambiado, particularmente entre los niños y jóvenes. Por lo general, podemos apreciar un creciente rechazo hacia la lectura (Postman, 1979). Otros autores establecen que el tiempo dedicado a ver televisión suplanta el que debería dedicarse a la lectura y al desarrollo de las habilidades lingüísticas (Neuman, 1988, Beentjes y Van der Voort, 1988).

Autores como Winn (1977), y Postman (1979), opinan que la televisión no favorece el desarrollo de la capacidad de atención en los niños, y esta capacidad es esencial en la adquisición de la lecto-escritura; sin embargo, no presentan pruebas empíricas al respecto. Bryant (1983), por su parte, sugiere que la televisión acostumbra a la mente del niño a las "tomas cortas", no a la continuidad del pensamiento que requiere la lectura.

Asimismo, algunos autores aseveran que las técnicas de entretenimiento fácil que despliega la televisión crean un obstáculo para la motivación a leer. El niño exige que el aprendizaje (de la lectura o de cualquier otra cosa) sea tan "movido" y divertido como la televisión, y esto, evidentemente, no siempre es posible (Fader, 1983; Singer y Singer, 1983; Postman, 1985)

Morgan y Gross, (1980), encontraron correlación entre consumo de televisión, baja habilidad de lectura y bajo cociente intelectual. Esto no sugiere, sin embargo, un modelo claro de causa y efecto. Al respecto anotan:

Los niffos que tienen problemas de lectura, o que ven la lectura como algo frustrante, intimidatorio, osimplemente aburrido, con frecuencia buscan estimulación a su fantasía y entretenimiento en la televisión. Aunque encontramos que la cantidad de horas pasadas frente al televisor predice las puntuaciones de lectura de un estudiante mejor que cualquier otra variable, la noción de que la televisión es la única causa no puede 
sostenerse, al menos en base a nuestros datos. La televisión puede con seguridad, reforzar un problema de lecturaya existente, en el sentidode que mientras un estudiante está mirando la televisión no puede concentrarse en la lectura. Incluso puede ser que un alto consumo de televisión no sea más que un signo sintomático del problema.

Los autores estadounidenses también han reportado una notable declinación en la circulación de libros entre los niños y jóvenes. Aún los niños que no tienen problemas con la lectura prefieren noleer, a menos que se les imponga como deber. El estilo de lectura de los niños actualmente también ha cambiado. Generalmente pasan las hojas y cambian de un libro a otro como buscando gratificaciones instantáneas similares a las que les da la televisión. Por otra parte, prefieren lecturas de baja calidad que tratan sobre sus héroes o monstruos televisivos. Esta literatura está escrita preferentemente en el lenguaje de la imagen, no de las palabras (Moody, 1980).

Aunado a esto, el lenguaje que el niño absorbe de los programas de televisión es mucho más simplificado que el de los libros. Estos presentan un vocabulario más amplio y usan con más frecuencia las estructuras gramaticales complejas. En cambio, la forma predominante del lenguaje en televisión es la "oración incompleta":

Los efectos aparentemente diferentes de la television y la lectura en el aprendizaje del lenguaje pueden estar relacionados a factores lingüísticos... el poner en contacto (al nifio) con el lenguaje complejo de los libros, que utilizan la mayorfa de las posibilidades sintácticas de la lengua, puede ayudarle a entender y usar el lenguaje más elaborado que tendrá que emplear en la escuela (Fasick, citada por Moody, 1980: 66).

Neuman (1988), examina las implicaciones del uso de la televisión para la competencia lectora y el desempeño escolar. Para ello utiliza la hipótesis del desplazamiento, la cual sugiere que ver televisión afecta el logro de lectura de los estudiantes al desplazar otras actividades cruciales para el desarrollo de las habilidades de lectura, como por ejemplo, la lectura por placer. En su trabajo, analiza cuatro teorías de desplazamiento: que la televisión desplaza las actividades funcionalmente similares; las actividades física y psicológicamente próximas; las actividades marginales; $y$ las actividades que se han vuelto menos atractivas debido a una reorganización de las necesidades funcionales que se da con la edad. 
Los resultados de su estudio indican que las diferencias en puntuaciones de lectura para los estudiantes que ven de dos a tres horas por día son pequeñas, pero que más de tres horas de exposición a la televisión trae consigo efectos negativos y crecientemente dañinos para la lectura.

Por otra parte, Beentjes y Van der Voort (1988) realizan una revisión de gran parte de la investigación empírica sobre la influencia de la television en las habilidades lectoras de los jóvenes, de 1954 a 1987. Describen en su trabajo las diferentes hipótesis que se han desarrollado para explicar por qué la televisión puede o no influir positiva o negativamente en las habilidades de lectura de los niños. Cada hipótesis revisada propone un mecanismo diferente a través del cual la televisión puede ejercer su influencia.

Ellos parten de una clasificación muy general que engloba la totalidad de las hipótesis manejadas en la literatura sobre el tema, desde los años cincuenta. Clasifican las hipótesis, grosso modo, en hipótesis de inhibición, de facilitación y de no efecto. Enseguida discutiremos brevemente los hallazgos que reportan bajo cada apartado.

\section{Hipótesis de inhibición}

Aquí se sitúan todos los estudios que tratan de establecer la forma en que la televisión actúa inhibiendo el desarrollo de las habilidades de lectura. Giran en torno a dos roles que se adjudican a la televisión: algunos estudios ven la televisión comouna actividad que consume tiempo, por loque puede desplazar las actividades que podrían ayudar al desarrollo de habilidades lectoras; otro grupo de hipótesis ven a la televisión como una experiencia de procesamiento de información, y se piensa que el niño que se expone continuamente a la televisión adquiere hábitos de procesamiento de información que interfieren con el desarrollo de las habilidades de lectura.

Las explicaciones sobre el procesamiento de la información recibida por un medio audiovisual como la televisión parten de dos asunciones:

a) que los niños procesan la información de la televisión más o menos automáticamente, sin hacer ningún esfuerzo mental. 
b) que el conocimiento de lo que se requiere para obtener información vía televisión se traslada y usa para procesar información de otra índole (por ejemplo la información que se obtiene por medios impresos).

\section{Hipótesis de facilitación}

Según estas hipótesis, la televisión promueve la lectura al ofrecer películas basadas en libros y programas que llaman la atención del auditorio hacia nuevas publicaciones. Y al aumentar la práctica de la lectura, sube la habilidad de leer. Lo que se ha sacado en claro sobre este punto, es que la mayoría de los estudiantes prefieren los libros que "vieron" por televisión (Hamilton, 1975; Splaine, 1978). Esto demuestra que la televisión puede fomentar la lectura de ciertos libros. Sin embargo, de aquí no se sigue necesariamente que la televisión induzca a leer más. Sólo parece redirigir la atención de lo que se lee.

Estas hipótesis también sostienen que la televisión puede mejorar la habilidad lectora en dos formas:

1) mediante programas educativos que buscan, específicamente, mejorar las habilidades de lectura; $y$

2) mediante la exposición a material escrito en la pantalla (créditos, avisos subtítulos, textos en los noticieros, etcétera).

Sobre lo primero no hay evidencia real positiva, pues los programas educativos sólo son eficaces si lo que pasa en la pantalla es más efectivo que lo que puede hacer un maestro en la escuela, y si hay apoyo (refuerzo) por parte de los padres.

Sobre el segundo punto, tal vez lo que pudiera ser más efectivo son los subtítulos de las películas, pues dan bastante oportunidad para practicar las habilidades de lectura.

\section{Hipótesis de cero efecto}

Esta hipótesis ofrece una explicación de por qué los investigadores no han tenido éxito en encontrar evidencias definitivas sobre la relación exposición a televisión y lectura. 
Una versión de esta hipótesis establece simplemente que tal relación no existe (Neuman,1986). Otra versión dice que tal vez los investigadores no han sidocapaces de detectar los efectos, que sí existen.(Neuman, 1980). Una tercera versión(Hornik, 1981) dice que la televisión sí influye en la lectura, pero mediante mecanismos que están funcionando con propósitos cruzados. Así, los efectos benéficos de la televisión se contrapondrían con los inhibitorios, y las habilidades de lectura no se verían alteradas.

Resumiendo, en esta revisión los autores encuentran poca evidencia sobre la validez de hipótesis explicatorias específicas, sin embargo, concluyen que existe más evidencia que sostiene las hipótesis de inhibición. Además, en el artículo se clarifican algunas condiciones bajo las cuales puede existir una relación entre televisión y logros de lectura, pues es claro que esta relación es mucho más compleja de lo que inicialmente se asumió.

Las condiciones que influyen tanto en la dirección como en la magnitud de la relación son las siguientes:

1. Tiempo de exposición: los estudios más recientes parecen indicar que la relación entre horas de exposición y logros de lectura no es una relación linear, sino curvilínea.

2. Tipo de programa al que se expone el sujeto: se ha visto mayor deterioro en habilidades de lectura en los que se exponen a programas de entretenimiento, y menor en aquellos que se exponen a programas de tipo informativo.

3. Estatus socioeconómico: La relación entre televisión ylectura es más negativa entre aquellos de alto estatus socioeconómico.

4. Inteligencia: Los sujetos con más alto cociente de inteligencia parecen ser los más perjudicados en sus habilidades de lectura al exponerse más a la televisión.

5. Género: La influencia negativa de la televisión en la lectura es mayor para las niñas. Se asume que la televisión desplaza cosas diferentes para cada sexo.

6. Edad: Aunque la evidencia no es concluyente parece haber más estudios que apoyan que la relación negativa entre televisión y lectura es más fuerte en los niños mayores. Gady (1986) explica que esto puede deberse a la "teoría del reto disminuido". Según éste, a medida que el niño crece, el contenido 
de la televisión ofrece menos estímulos intelectualmente retadores al niño, por lo que las posibles "ventajas" de la estimulación se pierden en los mayores.

7. Tipo de habilidades lectoras: La exposición desmedida a la televisión puede tener diferentes efectos dependiendo de la sub-habilidad de lectura que se trate. La mayoría de los estudios se orientan en la lectura de comprensión, pero también se pueden dar efectos en vocabulario o rapidez para descifrar.

Como hemos mostrado hasta ahora, la literatura extranjera sobre el tema se ha centrado principalmente en los efectos del uso de la televisión en la habilidad lectora (readingperformance) de niños (Neuman, 1988 y Beentjes, 1988), y no hemos encontrado estudios recientes sobre el tema enfocados hacia los jóvenes.

En México, si bien se han realizado estudios nacionales y locales sobre las deficiencias en habilidades lingüísticas entre jóvenes, parecen no existir investigaciones empíricas que se hayan centrado específicamente en determinar si puede darse y de qué manera una relación entre estas deficiencias y el uso de un medio audiovisual de tanta penetración como es la televisión (Véase Fuentes, 1988).

\section{La investigación}

Dada la ausencia de suficiente fundamentación empírica de investigaciones previas enfocadas a jóvenes en el tema que nos ocupa, aplicables a nuestro medio, el presente estudio tiene un carácter mayormente exploratorio y descriptivo, donde se intenta establecer primero los patrones de exposición a la televisión (en contraste con la exposición de varios medios impresos: periódico, revistas y libros), para luego ver si existe alguna relación entre estos patrones de exposición y la habilidad de razonamiento verbal en jóvenes de primer ingreso de una universidad privada.

Las variables dependientes e independientes que manejamos en este estudio se definen operacionalmente a continuación: 


\section{Variables independientes}

1. Exposición a televisión. Es el espacio temporal diario que el joven dedica a ver cualquier tipo de programa ofrecido en cualquier tipo de canal televisivo, o bien, grabado y reproducido en un aparato videoreproductor (VCR). Operacionalmente, se midió por horas al día, para obtener el estimado semanal de tiempo de exposición. Si bien, en el caso de niños menores de 10 años, los estimados en horas de tiempo dedicado a ver televisión suelen no ser muy confiables, existen estudios que apoyan el uso de este estimado en jóvenes y adultos, ya que en estos casos, el estimadó suele apegarse más a la realidad. (Anderson et al, 1985; Price et al. 1986; Vooijs, Van der voort y Beentjes, 1987).

2. Estrategias de procesamiento de información audiovisual. Es la forma en que los estímulos audiovisuales que proceden de la televisión son recibidos y procesados cognitivamente por el joven para generar sentido y obtener una recompensa (intelectual, sensorial o emotiva) de la exposición a la televisión. Se estableció mediante preguntas específicas para determinar el nivel de atención (actitud y actividad del niño mientras está viendo la televisión), la participación o actividad cognitiva que el joven despliega mientras recibe los mensajes televisivos, y las cualidades de los estímulos audiovisuales preferidas por el joven.

\section{Variables dependientes}

1. Habilidades de razonamiento verbal. Operacionalmente, se obtuvo por el puntaje de la prueba del College Board en la parte de razonamiento verbal.

2. Exposición a medios impresos. Se refiere tanto a la cantidad de tiempo, como a la frecuencia con que el joven se expone a diferentes medios impresos (en este caso, periódico, libros y revistas). Se midió mediante estimados autorreportados independientes para cada uno de los medios. En el caso del periódico, se tomó la frecuencia con que se lee (de cero a siete días a la semana) y las secciones que se leen. Para las revistas, se pidió que indicaran cuáles son las revistas que leen, y qué tiempo promedio a la semana dedican a su lectura. En cuanto a los libros, se anotó el número de horas a la semana 
que dedican a la lectura de libros, y la cantidad de ellos leída en el mes anterior. Se solicitaron también los nombres de los últimos libros leídos que recuerdan.

3. Gratificaciones asociadas a la lectura. Se midieron en cuanto a la satisfacción - positiva o negativa - que puede ofrecer la lectura de cada medio respecto de necesidades particulares del joven (aprender, distraerse, excitarse, como hábito, para relacionarse socialmente, como evasión).

Las relaciones entre las variables independientes y dependientes, se controlaron además por:

- Sexo. En estudios hechos en el extranjero se han encontrado evidencias de que la televisión desplaza cosas diferentes para niños y para niñas, haciendo que su influencia sobre las habilidades escolares sea más fuerte para las niñas (Williams et al,1982)

- Escuela de procedencia (bilingüe o monolingüe, privada o pública). Esta variable tampoco parece haber sido considerada en otros estudios, y en el nuestro consideramos necesario incluirla dado que en el medio de donde proceden los alumnos es común el haber realizado al menos una parte de sus estudios en una escuela bilingüe, lo cual puede ser una razón más para la pérdida de las habilidades verbales del español.

- Tipo de programas preferidos. Se ha encontrado que los programas de entretenimiento tienen un efecto negativo en las habilidades de lectura (Degrosky, 1981; Newman, 1981; Smyser-O'Sullivan, 1981), mientras que la relación es positiva cuando se trata de programas informativos o de noticias (Potter, 1987).

- Gratificación asociada a la exposición a televisión. Según algunos estudios, la gratificación obtenida por el uso de la televisión puede desplazar a la gratificación asociada con la lectura y escritura, así como otras actividades necesarias para el desarrollo de estas habilidades. (Neuman, 1988). 
Las preguntas de investigación

1. ¿A mayor tiempo de exposición a la televisión, existirá menor puntaje en habilidades de razonamiento verbal?

2. ¿A mayor exposición a la televisión, existirá mayor rechazo hacia la lectura? (en cuanto a tiempo dedicado a ella, frecuencia y gratificación asociada).

3. A mayor exposición a televisión, habrá menor exposición a medios impresos?

4. ¿Qué variables intervinientes podrían contribuir para que se den las relaciones entre exposición a televisión, exposición a medios impresos y habilidades de razonamiento verbal del joven?

\section{Metodología}

El universo del cual partimos fueron 320 alumnos de primer ingreso de licenciatura en la Universidad de Monterrey en enero de 1993. Esta población se caracteriza por ser bastante homogénea en cuanto a edad (17-18 años) y clase socioeconómica ( media-media alta). Existe también en ella equilibrio entre ambos sexos. Aunque de acuerdo con la bibliografía extranjera revisada ( $y$ al no encontrar bibliografía nacional al respecto) no hay evidencia de si el tipo de institución (pública o privada) introduce variaciones importantes a los resultados, asumimos que nuestros resultados no deberían generalizarse más que a alumnos de edad y nivel socioeconómico similares a los de este estudio.

La mayoría de estos alumnos ingresan por primera vez a una carrera profesional. Un pequeño número vienen de otras universidades, por cambio de carrera o residencia. Dada la ausencia de información sobre el posible comportamiento de este universo en cuanto a las variables que nos ocupan, asumiremos a efectos de muestreo un criterio conservador de $\mathrm{p}=0.50$ y $\mathrm{q}=0.50$, de manera que, para trabajar con un margen de error de 0.05 y un nivel de confianza de $95 \%$, el tamaño de la muestra, deberá ser de 173 casos.

Se utilizó un cuestionario para recoger información sobre los patrones de uso de televisión y de materiales escritos (libros, revistas, periódicos). La prueba del College Board se empleó para obtener los puntajes de razonamiento verbal en cada caso. 
El cuestionario fue estructurado con preguntas abiertas y cerradas, para establecer exposición a la televisión contra medios impresos, en cuanto a frecuencia y modalidad de exposición, así como las variables demográficas pertinentes (sexo, edad, escuela de procedencia), y la percepción familiar y personal hacia la lectura.

Los puntajes para determinar la habilidad de razonamientoverbal de los alumnos se obtuvieron del puntaje de la sección verbal de la Prueba de Aptitud Académica del College Board, que se aplicó a todos los aspirantes de nuevo ingreso a la Universidad de Monterrey (nuestra población) durante los meses de noviembre, diciembre (1992) y enero (1993). Elegimos esta opción ya que es una prueba validada para Latinoamérica, y mide la habilidad del muchacho para entender lo que se lee y la amplitud de su vocabulario.

Se usó un procedimiento de selección de muestra simple al azar. Del listado del total de alumnos de primer ingreso a la Universidad de Monterrey en licenciatura, se seleccionaron mediante una tabla de números aleatorios los casos necesarios según la cuota requerida para la muestra. Una vez obtenido el listado de la muestra, se localizó a cada uno de los alumnos mediante las listas de los grupos de primer semestre. Las encuestas fueron autoaplicadas, en las dos últimas semanas de mayo, en forma grupal.

Los datos del puntaje del College Board se ubicaron en la lista que nos proporcionó Dirección Escolar, para cada uno de los alumnos encuestados. Estos puntajes fueron obtenidos por los alumnos en el examen de admisión a la Universidad de Monterrey en los meses de noviembre 1992 a enero de 1993. Entre esta evaluación y el momento en que se aplicó el cuestionario para determinar los patrones de exposición a la televisión y medios impresos hay tres meses de diferencia, pero no consideramos que el tiempo transcurrido en este caso sea un factor que altere significativamente los datos de la evaluación del College Board, ya que las habilidad de razonamiento verbal no se modifican sustancialmente en tan poco tiempo.

\section{Limitaciones}

Entre las limitaciones de este estudio tenemos que la búsqueda bibliográfica parece indicar que no hay muchos estudios de este tipo realizados en el país, así que no nos podemos basar en indicadores o hallazgos previos válidos de correlaciones entre uso de televisión en jóvenes y habilidades verbales aplicables a nuestro medio. 
Se utilizaron, en algunos casos, y sólo como punto de referencia, los resultados reportados en el estudio de Fernández et al (1986), en cuanto a patrones de exposición a televisión en niños.

Segundo, que se aplicó la encuesta sólo a estudiantes de primer ingreso, en una universidad privada, por lo que no será generalizable más que para este tipo de estudiantes. Sin embargo, dado que es un campo donde parece ser no existe ningún indicador previo, el presente trabajo ofrece la posibilidad de un primer acercamiento exploratorio al tema, para que más adelante, si los resultados arrojan evidencias interesantes, pueda ser continuado de forma que permita generalizarse a una población más amplia.

Y por último, se usará como indicador de habilidad verbal el College Board, aunque no arroja evidencia sobre el desempeño de la habilidad verbal escrita del joven. Consideramos que esto no resta valor al estudio pues existen evidencias de que las habilidades de razonamiento verbal en general, están positivamente relacionadas con el desempeño por escrito.

\section{Resultados}

\section{Descripción de la muestra}

Ladistribución por sexoestá bastante equilibrada: $55.4 \%$ hombres y $44.6 \%$ mujeres, de clase media alta y alta, y de edades entre 16 y 17 años, de los cuales provienen de preparatoria privada e1 $90.9 \%$, y de preparatoria bilingüe el $28 \%$. Todos cursan el primer semestre de alguna de las 19 carreras en la Universidad de Monterrey.

Para contrastar las aseveraciones respecto de la pérdida de habilidades de razonamiento verbal sacamos la media en el puntaje del College Board, que para nuestra muestra es de 540 puntos, con una moda de 481 , una mediana de 528 y desviación estándar de 78.25.

Dado que carecemos de un puntaje local de contraste, nos limitaremos a observar que, en un reporte dado en 1992 por el Consejo Escolar de los Estados Unidos, la media en el puntaje de razonamiento verbal obtenido mediante la prueba SAT (que es una prueba similar a la del College Board), para los estudiantes que pidieron ingreso a universidades americanas fue en 1991 de 422, y en 1992 de 423, lo cual sitúa a los jovenes de nuestra muestra en una posición de bastante ventaja respecto de sus contrapartes estadounidenses. 
Hay que considerar, sin embargo, que el hecho de que nuestra muestra está compuesta en su mayoría por muchachos de clase social alta, que han tenidoacceso, en su mayoría, a una educación privada, y (supuestamente) de más calidad, no es de extrañar que tengan puntajes en general más altos. Aquí sería necesario tener acceso a los puntajes de jóvenes de otras clases sociales, y que pasaron la mayor parte de su erucación por escuelas públicas, para poder decir si en realidad los jóvenes mexicanos se manejan mejor en esta área.

Por otra parte, no es posible establecer si efectivamente existe una pérdida de habilidades verbales en los jóvenes mexicanos, sobre todo por el hecho de que no hay mediciones previas del razonamiento verbal de niños o jóvenes antes de que la televisión empezara a penetrar fuertemente en sus vidas.

Otro de los supuestos que manejan tanto autores extranjeros como mexicanos es que los niños y jóvenes han perdido el interés y gusto por la lectura, particularmente de libros.

Como podemos ver en la tabla 1, entre las preferencias de lectura, la menos favorecida es la de libros, manteniéndose bastante alta la inclinación hacia la lectura de revistas. Sin embargo, dado el tipo de revistas que más se leen (ver tabla 10) nos preguntamos si el alto contenido visual de las mismas es lo que les atrae, más que la lectura propiamente dicha de la parte verbal (artículos, notas).

Tabla 1. Frecuencia por medios impresos

\begin{tabular}{llcc}
\hline & & Si & No \\
\hline & Te gusta leer periodico & 79.6 & 20.4 \\
& Te gusta leer revistas & 86.7 & 13.3 \\
& Te gusta leer libros & 69.5 & 30.5 \\
\hline
\end{tabular}

\section{Patrones de uso de televisión y medios impresos}

En el estudio con niños del Distrito Federal realizado por Fernández Collado et al. (1986), se calculó un promedio de dos a tres horas diarias de exposición a televisión, y se supuso que a medida que el niño crece, el número de horas disminuiría, con base en la redistribución funcional de su tiempo.

Este número de horas - establecido para niños - coincide más o menos con el estimado por autores estadounidenses, japoneses y australianos, que reportan un 
promedio de 2 a 3 horas diarias (14 a 21 por semana). Nielsen (1987), encontró que el tiempo dedicado a ver televisión por las personas tiende a disminuir hacia la adolescencia ( $\mathrm{y}$ da un promedio de 20 horas semanales), para volver a repuntar, y alcanzar su grado máximo después de los 50 años, en que puede subir hasta 40 horas a la semana. Y Neuman (1990), en un estimado más conservador, establece que siendo realistas, probablemente los adolescentes consumen 10 horas semanales de televisión.

En nuestro estudio, se encontró que los jóvenes dedican un promedio de 2 a 2.5 horas diarias, y un promedio de 16 horas semanales a ver la televisión. El día en que menos ven la televisión es el sábado, posiblemente por ser un día usado preferentemente para sus actividades sociales. Como podemos ver, los jóvenes de esta muestra se comportan de manera similar a los de otros estudios extranjeros, en cuanto a horas de consumo de televisión.

La tabla siguiente muestra las horas por semana que los encuestados dijeron exponerse a la televisión.

Tabla 2. Exposición frente a la televisión

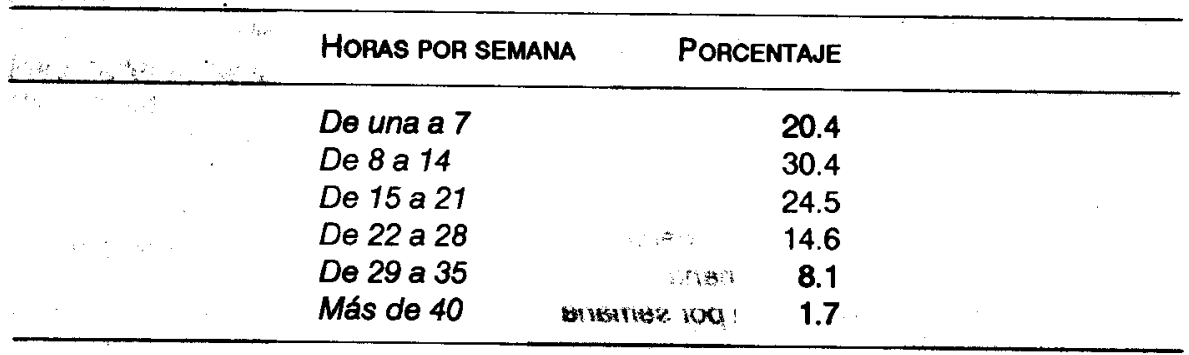

Exposición comparada, preferencias y gratificaciones de televisión y medios impresos

Si comparamos estimativamente la frecuencia de uso de cada medio, vemos que el tiempo semanal dedicado a ver televisión es mayor que el dedicado a cualquiera de los otros medios, particularmente la lectura de libros (tablas 2 a 5).

Las horas promedio dedicadas a ver televisión por semana son 15.5 , la frecuencia media de lectura de periódico es de 1 ó 2 veces por semana y la frecuencia media de lectura de revistas es de una cada 15 días. En cuanto a las horas dedicadas a 
lectura de libros los datos arrojaron una moda por semana de menos de 2 horas, y una media de 2 a 4 horas.

Para contrastar lo anterior se les preguntó por la actividad más reciente de lectura. Un $80.7 \%$ mencionó al menos una revista leída la semana anterior, un $65.5 \%$ recordó una noticia leída en ese mismo tiempo, y sólo un $34.5 \%$ nombró un libro leído en el mes anterior.

Tabla 3. Frecuencia de lectura de perib́dicos

\begin{tabular}{lr}
\hline Frecuencia & Porcentave \\
\hline Nunca & 4.1 \\
Raramente & 27.6 \\
162 veces por semana & 20.0 \\
3 o más veces por semana & 25.9 \\
Diariamente & 21.8
\end{tabular}

Tabla 4. Frecuencia de lectura de revistas

\begin{tabular}{lr}
\hline FreCUENCIA & POACENTANE \\
\hline Nunca & 2.4 \\
Raramente & 14.1 \\
Una al mes & 23.5 \\
Una cada quincena & 28.8 \\
Una por semana & 23.5 \\
Más de una por semana & 7.6
\end{tabular}

Tabla 5. Tiempo dedicado a leer libros por semana

\begin{tabular}{llr}
\hline & Horas a la semana & Porcentane \\
\hline & Menos de dos horas & 53.6 \\
De dos a seis horas & 30.4 \\
De siete a 14 horas & 9.5 \\
Más de 14 horas & 6.5
\end{tabular}


Una posible explicación del desplazamiento de la lectura en la vida del joven podría ser que la televisión, en particular, o los otros medios, cumplieran funciones similares, y que sustituyeran el uso de un medio como el libro por otro que ofrece la misma satisfacción funcional (Neuman, 1988).

Sin embargo, como vemos en las tablas 6 a 9, las gratificaciones que buscan en televisión no son las mismas de los libros, por lo que el desplazamiento no se podría explicar como desplazamiento por misma satisfacción funcional: los libros los leen principalmente para aprender, los diarios, para informarse, y las revistas, para divertirse y estar al tanto de novedades, mientras que la televisión es vista primordialmente como una forma de diversión y pasatiempo.

Tabla 6. Gratificaciones asociadas a la televisión

\begin{tabular}{lcccc}
\hline & MediA & ModA* & Mediana* & Desv.EsT. \\
\hline Aprender cosas nuevas & 2.08 & 2 & 2 & 0.62 \\
Por costumbre & 2.42 & 3 & 3 & 0.73 \\
Por experimentar emociones & 2.09 & 2 & 2 & 0.69 \\
Para evadirme & 2.29 & 3 & 2 & 0.70 \\
Para tener tema de conversación & 2.62 & 3 & 3 & 0.57 \\
Para matar el tiempo & 1.89 & 2 & 2 & 0.66 \\
Para divertirme & 1.71 & 2 & 2 & 0.68 \\
Reemplazo de interacción social & 2.74 & 3 & 3 & 0.53 \\
\hline
\end{tabular}

"En una escala donde 1 es "de acuerdo", 2 es "parcialmente de acuerdo y 3 es "En desacuerdo"

Tabla 7. Gratificaciones relacionadas con el periodico

\begin{tabular}{lr}
\hline RAzONES PARA LEER O NO El PERIódICO & PORCENTAJE \\
\hline Silo leo porque: & $(75 \%)$ \\
Me informa & 63.9 \\
Aprendo & 3.6 \\
Otras razones & 7.5 \\
No lo leo porque: & $(25 \%)$ \\
Me da sueño & 4.1 \\
Es más fácil enterarse por la televisión & 2.9 \\
Otras razones & 18.0
\end{tabular}


Tabla 8. Gratificaciones relacionadas con las revistas

\begin{tabular}{lr}
\hline RAzONES PaRA LEER O No ReVIstas & PORCENTANE \\
\hline S/ lo leo porque: & $(88 \%)$ \\
Me divierte & 14.5 \\
Me informa & 13.9 \\
Trae articulos interesantes & 12.3 \\
Trae chismes de artistas & 9.4 \\
Trae moda y tips de belleza & 8.8 \\
No lo leo porque: & $(12 \%)$ \\
Son poco confiables & 3.6 \\
Son aburridas & 2.4 \\
Otras razones & 6.0 \\
\hline
\end{tabular}

Tabla 9. Gratificaciones relacionadas con libros

\begin{tabular}{lr}
\hline RAZONES PARA LEER O NO LIBROS & PORCENTANE \\
\hline SI lo leo porque: & $(66 \%)$ \\
Aprendo & 32.5 \\
Me entretienen & 9.4 \\
Ayudan a mejorar redacción y vocabulario & 7.0 \\
Por otras razones & 17.1 \\
No lo leo porque: & $(34 \%)$ \\
Son tediosos & 20.0 \\
Me desespero & 5.0 \\
No me gusta leer & 3.8 \\
Por otras razones & 5.2 \\
\hline
\end{tabular}

Estas gratificaciones y satisfacciones funcionales se ven confirmadas por el tipo de contenidos que dijeron buscar en cada medio (ver tablas 10 a 13). Mientras que en revistas buscan aquellas con artículos interesantes (Selecciones del Reader's Digest) o con chismes de artistas (Eres y Somos), en el periodico buscan secciones que los mantienen informados de su entorno más próximo (el suplemento Sierra Madre es obligado entre estos jóvenes, seguido por la Sección Joven, Local y Moda). En televisión prefieren películas, programas cómicos, de aventura y drama - todos ellos dirigidos al mero entretenimiento - y en los libros recurren más a los textos de clase - para aprender. 
Tabla 10. Preferencia de lectura por tipo de revista

\begin{tabular}{lrrrr}
\hline Tipo o nombre de Revista & Media* & ModA & Mediana & Desv.Est. \\
\hline TVy novelas & 1.74 & 1 & 2 & 0.73 \\
Seventeen & 1.36 & 1 & 1 & 0.57 \\
Interview & 1.24 & 1 & 1 & 0.50 \\
Hola & 1.61 & 1 & 1 & 0.70 \\
Fotonovelas & 1.13 & 1 & 1 & 0.39 \\
Vanidades/Buenhogar & 1.46 & 1 & 1 & 0.63 \\
Eres/Somos & 2.26 & 3 & 2 & 0.75 \\
Proceso/Nexos & 1.40 & 1 & 1 & 0.61 \\
Tiempo/Newsweek & 1.43 & 1 & 1 & 0.63 \\
Elle/Mariclaire & 1.39 & 1 & 1 & 0.65 \\
Cosmopolitan & 1.53 & 1 & 1 & 0.65 \\
Revistas académicas & 1.48 & 1 & 1 & 0.61 \\
Selecciones del Reader's Digest & 1.98 & 2 & 2 & 0.70 \\
Nuevo foro/Nave & 1.53 & 1 & 1 & 0.75 \\
\hline
\end{tabular}

"En una escala donde 1 es "Nunca la leo", 2 es "A veces la leo" y 3 es "Siempre la leo".

Tabla 11. Preferencia de lectura por secciones del perib́dico

\begin{tabular}{llcccc}
\hline SEcción & & MEdiA & MOdA & MEDIANA & Desv.EsT. \\
\hline Internacional & 1.98 & 2 & 2 & 0.74 \\
Editorial & 1.54 & 1 & 1 & 0.64 \\
Local & 2.15 & 2 & 2 & 0.65 \\
Sociales & 2.46 & 3 & 3 & 0.70 \\
Cultural & 2.03 & 2 & 2 & 0.69 \\
Tiras cómicas & 1.82 & 2 & 2 & 0.75 \\
Negocios & 1.67 & 1 & 2 & 0.74 \\
Moda & 2.04 & 2 & 2 & 0.78 \\
Sección joven & 2.42 & 3 & 3 & 0.67 \\
Nota roja & 1.81 & 1 & 2 & 0.78 \\
Avisos de ocasión & 1.68 & 2 & 2 & 0.65 \\
\hline
\end{tabular}

"En una escala donde 1 es "Nunca la leo", 2 es "A veces la leo" y 3 es "Siempre la leo". 
Tabla 12. Preferencia por tipo de programas de televisión

\begin{tabular}{lcccc}
\hline & MEDIA $^{*}$ & MODA $^{*}$ & MEDIANA & DEsv.EsT. \\
\hline Deportivos & 2.07 & 2 & 2 & 0.65 \\
De concurso & 1.75 & 2 & 2 & 0.58 \\
Telenovelas & 1.85 & 2 & 2 & 0.66 \\
De opinión y debate & 2.15 & 2 & 2 & 0.57 \\
Aventura y drama & 2.48 & 3 & 3 & 0.57 \\
Noticieros & 2.03 & 2 & 2 & 0.63 \\
Cómicos & 2.48 & 3 & 3 & 0.57 \\
Videoclips & 2.38 & 3 & 2 & 0.64 \\
Caricaturas & 1.97 & 2 & 2 & 0.65 \\
Documentales & 2.03 & 2 & 2 & 0.70 \\
Pelicuias & 2.74 & 3 & 3 & 0.46 \\
\hline
\end{tabular}

"En una escala donde 1 es "Nunca los veo", 2 es "A veces los veo" y 3 es "Siempre los veo".

Tabla 13. Preferencia de lectura por tipo de libro

\begin{tabular}{|c|c|c|c|c|c|}
\hline TIPO DE LIBRO & & MEDIA & MOOA ${ }^{*}$ & MEDIANA" & " DEsv.Est. \\
\hline Ciencia ficción & & 1.70 & 2 & 2 & 0.78 \\
\hline Autobiográfico & & 1.55 & 1 & 1 & 0.63 \\
\hline Romance & & 1.62 & 1 & 1 & 0.71 \\
\hline Terror & & 1.56 & 1 & 1 & 0.69 \\
\hline Poesía & & 1.51 & 1 & 1 & 0.70 \\
\hline Históricos & & 1.71 & 1 & 2 & 0.71 \\
\hline Científicos & & 1.54 & 1 & 1 & 0.67 \\
\hline Literatura & & 1.57 & 1 & 1 & 0.74 \\
\hline Arte & & 1.63 & 1 & & 0.74 \\
\hline Cómicos & & 1.85 & 2 & 2 & 0.69 \\
\hline Aventura & & 1.93 & 2 & 2 & 0.71 \\
\hline Filosofía & & 1.33 & 1 & 1 & 0.59 \\
\hline Autosuperación & & 1.69 & 1 & 2 & 0.73 \\
\hline Textos de clase & $\because$ & 2.23 & 2 & 2 & 0.64 \\
\hline
\end{tabular}

"En una escala donde 1 es "Nunca lo leo", 2 es "A veces lo leo" y 3 es "Siempre lo leo". 


\section{Exposición a televisión y procesamiento de información}

Algunas de las hipótesis de inhibición sometidas a prueba por autores extranjeros en torno a la influencia de la televisión sobre el desempeño lector de niños, presuponen que el medio audiovisual tiene aspectos formales y una gramática particular que acostumbraría al niño a un estilo de procesamiento de información diferente de como se procesa la lectura, y que esto, a la larga, generaría un rechazo de esta última, por involucrar un esfuerzo mayor y menos gratificante a corto plazo (Hodges, 1986; Beentjes y Van der Voort, 1988).

Para ver cuáles de los aspectos formales más comunes de la imagen televisiva prefieren los jóvenes, se les cuestionó sobre los que aparecen en sus programas favoritos. Los resultados (ver tabla 14) muestran que entre las características que más buscan en los programas son los colores atractivos, formas novedosas, armónicas y llamativas, los diálogos breves y la música, y lo que menos les atrae son ángulos visuales novedosos, los diálogos largos y el texto en pantalla.

Si bien el hecho de que se prefieren los estímulos que son más exclusivos de la imagen visual, mientras que se rechazan aquellos que tienen más relación con lo verbal nos dice algo sobre una tendencia hacia lo analógico, que no involucra demasiado esfuerzo y que ofrece gratificaciones inmediatas, esto no asegura que esta inclinación tenga como efecto el rechazo a la lectura.

Tabla 14. Cualidades formales presentadas en

ition los programas de televisión que se prefieren ver

\begin{tabular}{lcccc}
\hline & MediA & MoOA & MedianA & Desv.Est. \\
\hline Ritmo acelerado de las escenas & 2.24 & 2 & 2 & 0.69 \\
Colores llamativos o agradables & 2.68 & 3 & 3 & 0.78 \\
Ángulos visuales novedosos & 2.20 & 2 & 2 & 0.89 \\
Imágenes que explican situaciones & 2.57 & 2 & 3 & 0.82 \\
Diálogos breves y concretos & 2.68 & 3 & 3 & 0.70 \\
Música & 2.94 & 3 & 3 & 0.73 \\
Diálogos largos & 2.20 & 2 & 2 & 0.73 \\
Texto en pantalla & 1.87 & 2 & 2 & 0.76 \\
\hline
\end{tabular}

"En una escala donde 1 es "Nunca", 2 es "A veces" y 3 es "Frecuentemente". y 4 es "Siempre". 
Al revisar caso por caso, las razones por las cuales los jóvenes que así lo reportaron no leen libros ( $y$ habría que tomar estas cifras con cautela, dado el contexto en que se puso la encuesta, y la clara conciencia de la "importancia de la lectura" que tiene la mayoría, aunque no la ejercite en la práctica), encontramos que aparte del simple y defensivo "porque no me gusta", se repiten las siguientes razones: "porque es tedioso, aburrido, me desespera, muy lento, tienes que esperarte muchas hojas para que pase algo, no son interesantes o atractivos, son largos, no son concretos, pierdo la atención, da flojera, son cansados, avanzas muy despacio, se cansa la vista, me hartan, da sueño, me duermen, prefiero algo más activo".

Estas razones podrían ser un indicio de que los adolescentes esperan de los medios impresos el mismo tipo de gratificación inmediata que ofrecen los medios audiovisuales, en donde no tienes que "leerte mil hojas para saber qué pasó con el personaje". Sin embargo al cruzar las variables de la tabla anterior con el número de horas dedicadas a la lectura por semana, encontramos que el único item que da resultados significantes a nivel de p. $=02$ es el de "diálogos breves y concretos", lo cual indica que quienes buscan principalmente estímulos visuales, con poca verbalización, en sus programas favoritos, dedican menos tiempo a la lectura de libros, donde los estímulos son cien por ciento verbales.

Tabla 15. Comparación entre el tiempo dedicado a la lectura de libros y la exposición a programas por diálogos breves y concretos

EXPOS ICION

TIEMPO dEDICAdo A LIBROS, POR SEMANA NUNCANA VeCES SIEMPAE TOTAL

Menos de dos horas

Dos a seis horas

Siete a 14 horas

Más de 14 horas
$34.4 \%$

$58.8 \%$

$33.3 \%$

$30.0 \%$
$65.6100 \%(n=90)$

$41.2100 \%(n=51)$

$66.7100 \%(n=15)$

$70.0 \quad 100 \%(n=10)$

$$
n=69 \quad n=97 \quad n=166
$$

$\times 2=9.10$

$g \mid=3$

significancia $p .=0.02$ 
Sin embargo este solo item no puede sostener toda la diversidad de supuestos referentes a que la televisión acostumbra al público joven a una sintaxis particular, y que esto los hace rechazar la verbal. Sería necesario repetir este tipo de pruebas, utilizando indicadores más afinados para establecer si la ausencia de resultados significativos se debe a una falla en la validez de los reactivos usados para medir los aspectos formales del mensaje televisivo preferidos por los jóvenes, o a una ausencia verdadera de relación entre éstos y la actitud hacia la lectura.

Relación entre medios impresos, televisión y puntaje en razonamiento verbal

Una vez realizada a grosso modo la exploración descriptiva de algunos de los patrones de exposición a medios, podemos decir que a nivel de la muestra, sí existe una tendencia a preferir la televisión frente a los medios impresos, particularmente ellibro. Sin embargo, no podemos afirmar que - al menos para nuestra población este aparente desplazamiento de la lectura tenga como causa la exposición desmedida a televisión.

Si vemos las tablas 16,17 y 18 , encontramos que de acuerdo con el análisis de varianza no se encontraron diferencias significativas entre las medias de horas de consumode televisión de los diferentes grupos de frecuencias de consumo a medios impresos.

Tabla 16. Tiempo de exposición a televisión, por semana y tiempo dedicado por semana a leer libros

(análisis de varianza simple)

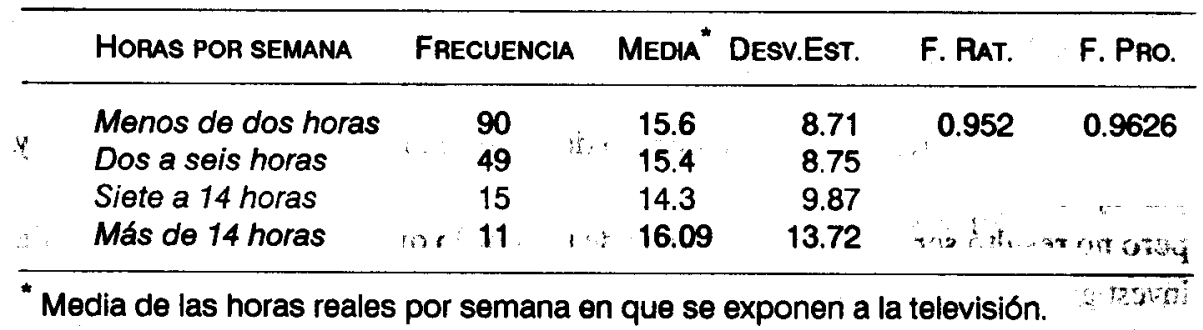


Tabla 17. Tiempo de exposición a la televisión por semana y frecuencia en la lectura de periódicos

(análisis de varianza simple)

\begin{tabular}{lcrrrr}
\hline Frecuencla de lectura & Frecuencia & MediA & Desv.Est. & F. Rat. & F. Pro. \\
\hline Nunca & 6 & 9.4 & 6.10 & 1.528 & 0.1962 \\
Raramente & 46 & 16.9 & 8.86 & & \\
Una o 2 veces por semana & 34 & 15.3 & 9.58 & & \\
3 a 6 veces por semana & 44 & 13.8 & 8.21 & & \\
Diariamente & 36 & 16.9 & 10.33 & & \\
\hline
\end{tabular}

" Media de las horas reales por semana en que se exponen a la televisión.

Tabla 18. Tiempo de exposición a la televisión por semana y frecuencia en la lectura de revistas (análisis de varianza simple)

\begin{tabular}{lcrrrr}
\hline Frecuencia de lectura & Frecuencia & Media & Desv.Est. & F. Rat. & F. Pro. \\
\hline Nunca & 4 & 10.3 & 8.55 & 1.346 & 0.2477 \\
Raramente & 23 & 18.1 & 9.16 & & \\
Una vez al mes & 40 & 12.9 & 8.71 & & \\
Una vez por quincena & 47 & 16.2 & 8.51 & & \\
Una vez por semana & 40 & 15.9 & 8.61 & & \\
Dos o más por semana & 13 & 15.7 & 13.40 & & \\
\hline
\end{tabular}

" Media de las horas reales por semana en que se exponen a la televisión.

Variables intervinientes en la relación entre televisión y razonamiento verbal

En cuanto a la correlación entre tiempo de exposición por semana a televisión y puntaje en razonamiento verbal, se obtuvo un coeficiente $r$ de Pearson de -.1332, pero no resultó ser significante al nivel de.01, por lo que la pregunta principal de investigación no se ve confirmada.

Dado que otros estudios extranjeros que no han encontrado una correlación significativa entre estas dos variables, han logrado algunas veces, controlando por 
una tercera variable, establecer al fin la correlación, decidimos controlar, de acuerdo con lo establecido en la definición de variables, por tipo de programa al que se exponen con más frecuencia, por sexo y por preparatoria de procedencia (monolingüe o bilingüe). Los resultados se muestran en las tablas 19 y 20.

Tabla 19. Tiempo de exposición a televisión, y puntaje de razonamiento verbal, controlado por sexo y escuela de procedencia

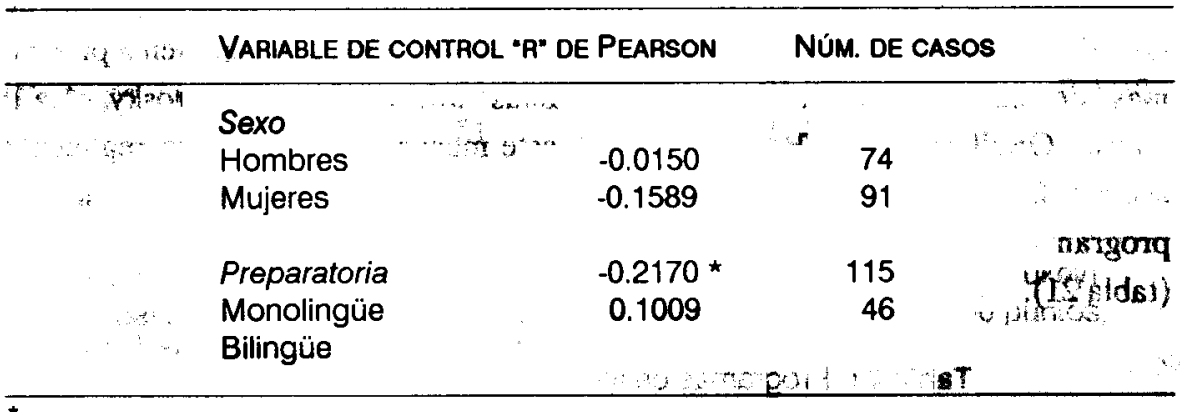

" Correlación significativa al nivel de $p=0.01$.

Tabla 20. Porcentaje de la prueba de razonamiento verbal y tipo de programa que ven con más frecuencia ("r" de Pearson)

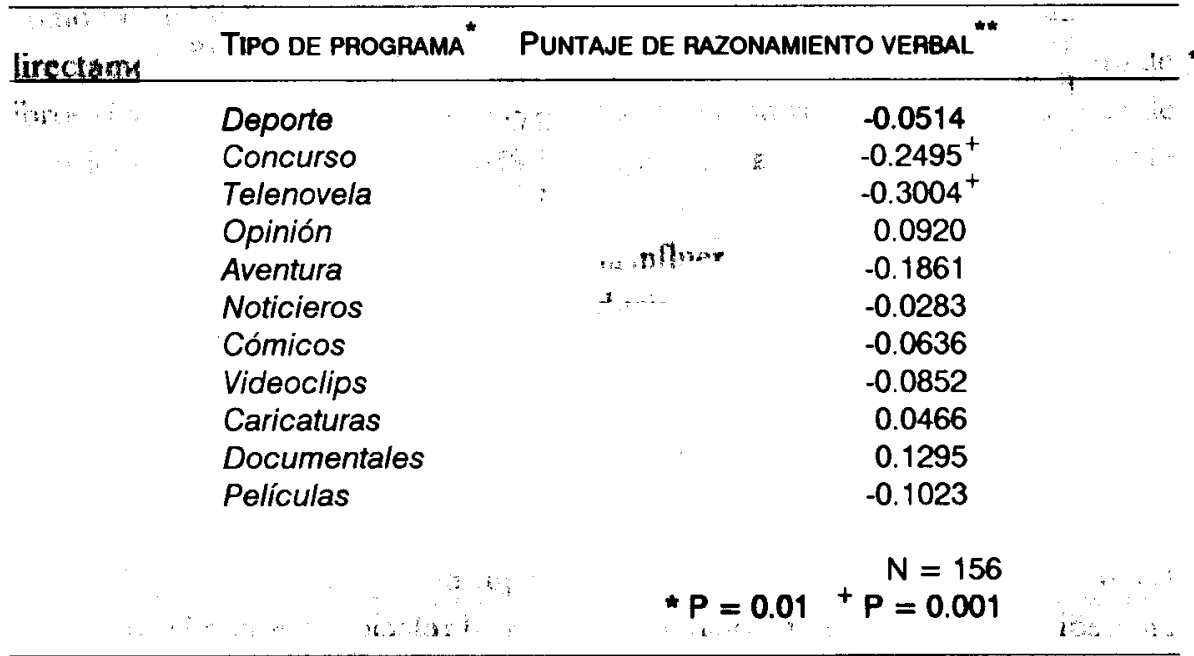

"Medido en la escala: 1 "Nunca los veo", 2 "A veces los veo" y 3 "Siempre los veo". ** Medido en números reales, donde 200 es el menor puntaje, y 800 el mayor. 
Sólo encontramos relaciones significativas si controlamos por preparatoria de procedencia y por tipo de programa al que se exponen.

Una posible explicación del mecanismo que actuaría en estos dos casos sería, para el primero de ellos, el hecho de que las preparatorias bilingües tal vez ofrezcan una mayor calidad en educación, lo cual repercutiría en todas las áreas del aprendizaje. En cuanto al tipo de programa, la relación encontrada va de acuerdo con los hallazgos de otros autores respecto de que la influencia de la televisión en las habilidades verbales parece ser mayor cuando los sujetos se exponen a programas de entretenimiento que a programas informativos (Degrosky, 1981; Smyser-OSullivan, 1981; Potter, 1987). Si este mecanismo funcionara realmente así, habría que prestar atención al hecho de que la mayoría de los jóvenes marcó programas de entretenimiento entre las primeras tres opciones de sus favoritos (tabla 21).

Tabla 21. Programas de televisión que más le gusta ver

\begin{tabular}{|c|c|c|c|}
\hline \multirow[b]{2}{*}{ NOMBRE DEL PROGRAMA } & \multicolumn{3}{|c|}{ Lugar de preferencia en que lo nombro } \\
\hline & Primero & SEGUNDO & TERCERO \\
\hline Beberly Hills 90210 & $30.5 \%$ & $6.7 \%$ & $6.9 \%$ \\
\hline Películas & $6.7 \%$ & $6.7 \%$ & $6.3 \%$ \\
\hline La cosa & $4.3 \%$ & $4.2 \%$ & $5.0 \%$ \\
\hline Telenovelas & $6.1 \%$ & $3.0 \%$ & $2.5 \%$ \\
\hline Futbol & $3.0 \%$ & $1.2 \%$ & $1.9 \%$ \\
\hline Los Simpson & $3.0 \%$ & $1.2 \%$ & - \\
\hline Guardianes de la bahia & $1.2 \%$ & $7.3 \%$ & $2.5 \%$ \\
\hline Cristina & $1.2 \%$ & $5.5 \%$ & $3.8 \%$ \\
\hline Viajeros en el tiempo & $0.6 \%$ & $4.8 \%$ & $1.9 \%$ \\
\hline Papá soltero & $0.6 \%$ & $4.2 \%$ & $3.1 \%$ \\
\hline Noticieros & - & $1.2 \%$ & $1.9 \%$ \\
\hline No contest6 & $8.5 \%$ & $15.2 \%$ & $24.5 \%$ \\
\hline
\end{tabular}

Por último, de los medios analizados, el único que mostró diferencias significativas en medias del puntaje de razonamiento verbal al relacionarlas con la frecuencia de exposición, fue el libro (ver tabla 22). Las diferencias aparecen entre los que leen menos de dos horas frente a quienes leen más de siete horas por semana. Esto 
parece indicar que ni el periódico ni las revistas favorecen el tipo de práctica de lectura que fomenta un mejor desarrollo de las habilidades de razonamientoverbal.

Tabla 22. Puntaje de razonamiento verbal y tiempo dedicado a lectura de libros por semana (análisis de varianza simple)

\begin{tabular}{lccccc}
\hline Lectura en la semana & Frecuencia & Media $^{*}$ Desv.Est. & F. Rat. & F. Pro. \\
\hline Menos de dos horas & 90 & $526.78^{\mathrm{a}}$ & 71.37 & 4.468 & $\mathbf{0} 0048$ \\
Dos a seis horas & 51 & 537.60 & 81.28 & & \\
Siete a 14 horas & 16 & $586.68^{\mathrm{b}}$ & 77.49 & & \\
Más de 14 horas & 11 & $587.72^{\mathrm{b}}$ & 74.15 & & \\
\hline
\end{tabular}

Medias con diferente superscript son significativamente diferentes (b de Tukey) "Media del puntaje de razonamiento verbal, en una escala de 200 a 800 puntos, donde 200 es lo más bajo y 800 lo más alto.

\section{Televisión y actitudes frente} al uso de la lectura en el encuadre escolar

Como ya vimos anteriormente, la sola exposición a televisión no está relacionada directamente con la habilidad de razonamiento verbal, mientras que la lectura de libros sí se relaciona positivamente con ella. También vimos que la frecuencia de exposición a libros es menor que a televisión, aunque no están directa o linealmente relacionadas.

La pregunta a responder ahora sería si la influencia de la exposición a televisión se podría dar de manera indirecta, al reducir el interés y gusto del joven por la lectura y actividades relacionadas con ella en el medio escolar, que es en donde existe mayor posibilidad de que el muchacho utilice un medio como el libro.

No se encontraron diferencias significativas por la relación entre tiempo de exposición a televisión y gusto por la lectura, gusto por la escritura y gusto por la investigación bibliográfica. La única diferencia significativa se encontró en horas de exposición a televisión y el gusto por el uso de materiales bibliográficos para preparar clase (tabla 23).

El hecho de que sólo una de las cuatro relaciones haya arrojado resultados significativos parece extraño, pero tal vez podría explicarse (arriesgando una 
aseveración a partir de mera observación personal) por el hecho de que, al menos en el medio en que fue levantada la encuesta, en la mayoría de las clases no se realizan actividades que involucran lectura, escritura, o verdadera investigación bibliográfica. En cambio, es más comán que el maestro pida a los alumnos que ellos preparen clase, lo cual implica leer algún capítulo de un libro, por lo que ésta es una experiencia que los alumnos sí conocen, y que por lo general les disgusta.

Tabla 23. Tiempo de exposición a televisión por semana y actitud frente al uso de material bibliográfico en clase

(análisis de varianza)

\begin{tabular}{lccrrr}
\hline Actitud frente al uso & Frecuencia & Media & Desv. Est. & F. Rat. & F. Pro. \\
\hline Me gusta mucho & 47 & $13.86^{\mathrm{a}}$ & 9.12 & 3.696 & 0.0269 \\
Me gusta poco & 81 & $14.71^{\mathrm{a}}$ & 8.34 & & \\
No me gusta & 40 & $18.76^{\mathrm{b}}$ & 10.14 & & \\
\hline
\end{tabular}

(Los grupos con diferente superscript son significativamente diferentes al nivel de $p$ $=0.05$, según la prueba $b$ de Tukey)

"Media de las horas reales por semana en que se exponen a la televisión.

\section{Conclusiones}

En este estudio hemos realizado un primer acercamiento exploratorio y descriptivo para establecer si existe relación entre habilidades de razonamiento verbal y uso de televisión, y entre habilidades de razonamiento verbal y uso de tres medios impresos (periódico, revista y libro). Las conclusiones fueron las siguientes:

1) No se encontró relación significativa (al nivel de $p=0.05$ ) entre tiempo de exposición a televisión (horas por semana) y razonamiento verbal (medido por el puntaje obtenido en la prueba del College Board).

2) No se encontró relación significativa entre tiempo de exposición a televisión y rechazo a la lectura, en cuanto a tiempo dedicado a ella y actitud (gusto por la lectura, por la escritura y por la investigación bibliográfica). Sólo se encontró relación significativa entre tiempo de exposición a televisión y actitud frente al uso de material bibliográfico para preparar clase. 
3) A pesar de que sí hay menor exposición a medios impresos, y particularmente a libros, que exposición a televisión, no se encontró ninguna relación inversa significativa entre el tiempo de exposición a televisión y el tiempo de exposición a medios impresos.

Tampoco se puede hablar de desplazamiento funcional (según lo previsto por Neuman, 1987) dado que las gratificaciones funcionales adjudicadas a cada medio que encontramos para esta muestra son diferentes, particularmente entre la televisión y los medios impresos revisados.

Si bien resultados como los obtenidos para estos tres primeros incisos concuerdan con los obtenidos por varios autores, que Beentjes y Van der Voort (1988) agrupan bajo el epígrafe de hipótesis de cero efecto, también hay otros estudios que sí han encontrado una relación significativa (Neuman, 1991), de manera que no existe aún una respuesta definitiva a la interrogante de si existe o no una relación entre habilidades verbales y uso de televisión.

Uno de los problemas, según la bibliografía revisada, es que la mayoría de los autores interesados en el tema han empleado indicadores diferentes, tanto para medir el tiempo de exposición como para medir las habilidades verbales (algunos usan comprensión de lectura, otros amplitud de vocabulario, calificaciones escolares, estimados de maestros, etc.). Algunos, incluso, no ofrecen claramente evidencias de significación estadística de sus resultados, o argumentan que la consistencia, por ejemplo, en relaciones débiles pero negativas, tiene más valor que su significancia estadística. Esto apunta hacia la necesidad de desarrollar instrumentos validados para la medición de estas variables, y hacia la importancia de repetir los estudios y usar metodologías de análisis y diseños de investigación más adecuados. En nuestro país, este es un campo de investigación aún casi sin explotar, lo cual permitiría establecer, desde un principio, líneas más coherentes de exploración, tomando en cuenta los resultados de las investigaciones ya realizadas.

Los hallazgos en diversos estudios, particularmente la revisión exhaustiva del tema que hace Neuman (1987), inclinan a pensar que posiblemente no se trata, en sentido estricto, de una relación directa y linear, sino de una relación curvilinear, que requeriría de metodologías más sofisticadas para ser detectada, ya que los efectos de la televisión no se presentarían sino a 
partir de cierto rango de exposición al medio, que en este caso, de acuerdo con los resultados revisados por Neuman, sería de más de cuatro horas diarias.

Otros autores han subrayado también la importancia de llevar a cabo estudios panel longitudinales, pues el impacto de la televisión podría hacerse evidente con más claridad de manera acumulativa (Koolstra y Van der Voort, 1990 y 1991).

Por otra parte, el hecho de que la mayoría de los estudios se han realizado con niños (generalmente menores de 12 años), nos lleva a suponer que si existiera una relación inversa entre exposición a televisión y desarrollo de habilidades verbales, ésta se haría más evidente en el tiempo en que se da mayor consumo de televisión, o sea, antes de la adolescencia. Como vimos en la discusión teórica, el número de horas que se dedican a ver televisión decrece con la edad, hasta llegar a la juventud, de manera que en los adolescentes puede no ser tan importante si se ve mucha o poca televisión, sino cuánta se vio en la niñez, mientras se desarrollaban las principales habilidades verbales y las actitudes hacia la lectura. Esto podría explicar la ausencia de relación encontrada en nuestros resultados.

4) Por lo que toca a las variables intervinientes, sólo el tipo de programa y la escuela de procedencia arrojaron diferencias significativas en cuanto a la relación entre horas de exposición a televisión y puntaje de razonamiento verbal. Contrario a lo que se esperaría, el hecho de asistir a una escuela bilingüe parece proteger a los jóvenes contra el impacto de la exposición a televisión en las habilidades verbales (del español, por supuesto), al menos en la población de donde fue tomada la muestra. No existe ninguna explicación teórica disponible que dé cuenta de esta relación.

En cuanto al tipo de programa, los estudios de Degrosky (1981) y Potter (1987) mostraron que la frecuencia de lectura se relacionaba negativamente a la exposición a programas de entretenimiento, y positivamente a la exposición a programas informativos y documentales. En nuestro estudio, los resultados que obtuvimos concuerdan con estos hallazgos, específicamente, en lo que concierne a los programas de concurso y telenovelas, que correlacionaron negativamente con el puntaje de razonamiento verbal. 
Sibien los resultados del presente estudio sostienen la hipótesis de que la exposición a televisión no tiene ninguna relación con las habilidades de razonamiento verbal en nuestra población, sería conveniente ampliar la investigación a otro tipo de poblaciones, por ejemplo, alumnos de universidades públicas, y jóvenes que trabajan en lugar de asistir a la universidad. También hace falta llevar a cabo estudios con niños en esta área, preferentemente mediante diseños panel, que permitan observar el impacto acumulado de la exposición a televisión en las habilidades verbales. Convendría asimismo el uso de herramientas de análisis más elaboradas, que permitan determinar relaciones no lineares, que en este estudio no se emplearon.

Por último, hay que anotar que las habilidades verbales son algo más complejo que el resultado de una prueba de razonamiento verbal, y aunque generalmente correlacionan positivamente con él, podrían ser completadas mediante otros parámetros, como comprensión de lectura, habilidad de expresión escrita, manejo de vocabulario, etc.

La investigación etnográfica, aunque más difícil de controlar y problemática (Neuman presenta un breve reporte de este tipo de estudios en su trabajo de 1986), podría también ser una fuente más de información que nos proporcionaría evidencias que escapan a los diseños de investigación cuantitativos, respecto de la relación de medios audiovisuales y habilidades de lecto-escritura y razonamiento verbal, sobre la cual no se ha dicho aún la última palabra.

\section{Referencias bibliográficas}

A. C. Nielsen Company (1987): 1987 Nielsen report on television. Northbrook, Illinois: Autor.

ANDERSON D. R. et al. (1985): "Estimates of young childrens time with television: A methodological comparison of parent reports with timelapse video home observation". Child Development 56, 1345-1357.

ARENAS Pedro (s.f.): La televisión y nuestra conducta cotidiana: efectos sobre los niños. Buenos Aires: Editorial Cuarto Mundo. 
ARMSTRONG G. B. y B. S. GREENBERG (1990): "Background television as an inhibitor of cognitive processing". Human Communication Research Vol. 16, Núm. 3, pp. 355-386 (primavera).

BASAVE P. (1980): Investigación sobre las habilidades verbales de estudiantes de la Universidad Regiomontana. (fotocopias)

BEENTJESJ. W.J.yTom VANDER VOORT (1988): “Televisions impact on childrens reading skills: a review of research". Reading Research Quarterly Vol. 23, Núm. 4, pp. 389-413 (otoño).

BETTELHEIM B. (1986): Aprender a leer. México: CNCA.

BRYANT Jennings, y D. ANDERSON (Eds.) (1983): Children Understanding of TV. Research on attention and comprehension. New York: Academic Press.

Charles M. (1986): "La televisión y los niños (el reto de vencer al Capitán América". Ponencia en el Coloquio La Televisión y el Niño, Morelia, agosto 1986.

CHÁVEZ G. et al. (1985): "Funciones de la televisión escolar". Televisión educativa en México, pp. 87-90. México: COSNET/SEP.

CORONA S. (1986): "La televisión y el niño. Una relación de doble apropiación (el caso de los superamigos)". Ponencia en el Coloquio La Televisión y el Niño, Morelia, agosto 1986.

Cremoux Raúl (1968): La TVy el alumno de secundaria del D. $F_{2}$ México: Centro de Estudios Educativos.

CROvi Delia (1985): "Lo espectacular de la televisión". La Televisión educativa en México, pp. 47-57. México: COSNET/SEP.

CullingFord Cedric (1984): Children and television. New York: St. Martin's Press. 
De la PeNa R. y R. Toledo (1992): "Consumo televisivo en el valle de México". Intermedios Núm. 3, pp.49-57 (agosto).

DORR A. y D. KUNKEL (1990): "Children and the media environment. Change and constancy amid change". Communication Research Vol.17, Núm. 1, pp. 5-25 (febrero).

ELLIOT N. (1979): "Language and cognition in the developing child". En E. WARTElla (ed.) Children Communicating pp. 187-215. Newbury Park, CA:Sage Publications.

Entrevista con Dora Antinori (1989): Monterrey, México.

FADER D. (1983): "Literacy and family". En R. BAILEY y R. FOSHEIM (eds.) Literacy for life, pp. 236-247. Nueva York: Modern Language Association.

FernándeZ Collado Carlos, Pilar BAPTiSTA Lucio y Débora Elkes (1986): La televisión y el niño. México: Oasis.

FerReiro E. y M. Gómez PALACIo (Comps.) (1991): Nuevas perspectivas sobre los procesos de lectura y escritura. México: Siglo XXI Eds.

FuENTES R. (1988): La Investigación de Comunicación en México. Sistematización documental, 1956-1986. México: Ediciones de comunicación.

GATTENGo Caleb (1973): Hacia una cultura visual. México: SEP, Col. Setentas.

GONZÁLEZ R. M. (1986): “Nuestros jóvenes no saben redactar”..Selecciones, pp. 46-50 (septiembre).

GREENFIELD Patricia Marks (1984): Mind and Media. The effects of TV, video games and computers. Cambridge, Mass.: Harvard Univesity Press.

Hamilton H. (1975): "TV tie-ins as a bridge to books". Language Ants Núm. 33, pp. 129-130. 
HoDGE Robert, y D. TRIPP (1986): Children and TV.A semiotic approach. Stanford, CA. : Stanford University Press.

HOMIK R. (1981): "Out of school television and schooling: hypoteses and methods". Review of Educational Research Núm. 51, pp. 193-214.

HowITT Dennis(1976): "The effects of TV on children". Ray BROWN(ed.) Children and $T V$, pp. 320-340. Beverly Hills: Sage.

JACOBY J. y W. D. HOYER (1989): "The comprehension/miscomprehension of print communication: selected findings".Joumal of Consumer Research Vol. 15, Núm. 4, pp. 434-433 (marzo).

KOOLSTRA Cees y Tom VAN DER VOORT (1992): Influence of television on childrens book reading. Conferencia presentada en el XX Congreso IAMCR, Guarujá, S. P. agosto 1992.

KRANSKY-BROWN L. (1986): "No, but I saw the movie". En L. KRANSKY-BROWN (ed.) Taking advantage of media, pp. 66-82. Boston, Mass.: Routledge \& Kegan Paul.

KUBEY R. y R. LARSON (1990): "The use and experience of the new video media among children and young adolescents". Communication Research Vol. 17, Núm.1, pp.107-130 (febrero).

LESSER Gerald (1974): Children and TV. Lessons from Sesame Street. New York: Vantage Press.

LIEBERT R. y J. SPRAFKIN (1988): The early window. Nueva York: Pergamon Press.

MCLUHAN Marshall (s.f.): "Electrónica y desenganche psicológico". En A. GRAS (ed.) Sociología de la Educación, pp. 75-79. Madrid: Ed. Narcea. 
MEDina J. (1986): "Panorámica de la investigación sobre los efectos de los contenidos no didácticos de los medios de comunicación social en México". Medios de Comunicación, Hegemonía y Proyectos de desarrollo, Cuadernos de Trabajo Núm. 1. México: AMIC/TICOM, UAM-X. México

MiNTON J. H. (1972): The impact of Sesame Street on reading readiness of kindergarden children. Disertación doctoral, Fordham University, Nueva York.

MontoyA A. y A. RebeiL (1983): El impacto educativo de la televisión en los estudiantes del Sistema Nacional de Telesecundaria .Cuadernos del Ticom, Núm. 28 (noviembre).

MoODY Kate (1980): Growing up on TV. The TV effect. New York: Times Books.

Morgan M. y L. Gross (1980): "Television viewing, IQ, and academic achievment". Joumal of Broadcasting Vol. 24, Núm 2, pp. 117-132.

Neuman S. B. (1991): Literacy in the television age. New Jersey: Ablex Publ. Co.

(1988): "The displacement effect: assesing the relation between television viewing and reading performance". Reading Research Quarterly, Vol. 23, Núm. 4, pp. 414-440 (otoño).

(1986): "Television, reading and the home environment". Reading Reserach and Instruction, 25, 173-183.

(1981): The effects of television on reading behavior. Willimantic: Eastern Connecticut State College.

(1980): “Why children read: a functional approach". Joumal of Reading Behavior 12, pp. 333-336.

NOBLE Grant (1975): Children in front of the small screen, Constable, London. Sage, Beverly Hills. 
OROzco G. (1985): “Apuntes para un debate sobre el impacto cognoscitivo de la televisión”. Renglones Núm. 3 (octubre). Guadalajara: IrESO.

(1986): "El estudio de los efectos educativos de la televisión en los niños. Algunos límites de la investigación tradicional". Ponencia presentada en el Coloquio La Televisión y el Niño, Morelia, agosto.

PATTON J., T. STINARD y D. RouTH (1983): "Where do children study?". Joumal of Educational Research Núm. 76, pp. 280-286.

PIAGET Jean (1972): El lenguaje y el pensamiento en el niño pequeño. Buenos Aires: Ediciones Guadalupe.

PoSTMAN N. (1985): Amusing ourselves to death. Nueva York: Vicking.

† (1979): Teaching as a conserving activity. New York: Delacorte Press.

POTTER W.J. (1987): “Does television viewing hinder academic achievment among adolescents?". Human Communication Research Núm. 14, pp. 27-46.

PRICE V. et al. (1986): The Stanford reading and television study, a progress report. Stanford, CA.: Institute for Communication Research. (ERIC Document Reproduction Service No. ED 277 064)

REBeIL M. (1986): Televisión infantil, investigación y acción civil. Ponencia presentada en el Coloquio La Televisión y el Niño, Morelia, agosto.

ROBERTS R. (1987): "Those who do not watch television". Sociology and Social Research. Vol. 71, Núm. 2, pp. 105-107 (enero).

RoDrfauez A. (1990): "Análisis de ciertos fenómenos lingüísticos en el habla de los universitarios y su repercusión social”. Monterrey: UANL.

RoE K. (1989): "School achievement, self steem and adolescent video use". En M. R. LEVY (ed.) The VCR age: home video and mass communication. Newbury Park, CA: Sage Publications. pp. 168-187. 
RoLANDELLI D.(1989): "Children and television: the visual superiority effect reconsidered". Joumal of Broadcasting and Electronic Media Vol. 33 Núm. 1, pp. 69-81 (invierno).

SALOMON G. (1979): "Shape, not only content: how media symbols partake in the development of abilities". En E. WARTELLA (ed.) Children Communicating. Newbury Park CA.: Sage Publications.

(1990): "Cognitive effects with and of computer technology". Communication Research Vol. 17, Núm. 1, pp. 26-44 (febrero).

SÁNCHEZ RUIZ E. (1989): "Televisión y representaciones políticas de escolares tapatíos". Comunicación y Sociedad Núm. 7, pp. 55-73.

SCHRAMM W., J. LyLE y E. PARKER (s.f.): Television in the lives of our children. Stanford, CA.: Stanford University Press.

SHOAMAKER P.J.(1989): "Predicting media uses".EnF.WILliams(ed.) Measuring the information society. Newbury Park, C A: Sage Publications, Sage Focus Editions, Núm. 97, pp. 229-242.

SINGER y SINGER (1983): Implications of Childhood TV viewing for cognition, imagination and emotion. Yale University Press.

SNOW R. P. (1988): "Forms, Formats and grammatical structure in mass media". En D. R. MMAINES y C. J. COUCH (eds.) Communication and social structure, pp. 201-214. Springfield, Il: Charles C. Thomas.

Television and growing up: the impact of televised violence (1971): Report to the Surgeon general, United States Public Health Service. Washington D. C.: US Government Printing Office.

VAN PEER W. (1988): "Reading culture and modern mass media". Joumal of Information Science Vol.14 Núm.5, pp. 305-309. 
Exposición a televisión, uso de medios impresos y razonamiento verbal entre jóvenes...

Voous M. W. et al. (1987): "Validity of self report measures of children's television viewing and reading time". Massacommunicatie Núm.15, pp.65-80.

WADSWORTH A.J. (1989): "The uses and effects of mass communications during childhood". En J. F. NUSSBAUM (ed.) Life-span communication normative processes, pp.93-116. Hillsdale, N. J.: Lawrence Erlbaum Associates.

WAGNER Daniel (ed.) (1987): The future of literacy in a changing world, Vol 1. New York Pergamon Press.

WILLIAMS Haertel, Haertel and Walberg (1982): "The impact of leisure time television on school learning". American Educational Research Joumal Núm.19, pp.19-50.

WINICX Marian. P. (1982): Children and TV. Mediating the medium. Washington.

WINN Marie (1977): The Plug-in Drug- TV and Family. Nueva York: Vicking Press.

WOBER J.M. (1990): "Never mind the picture, sense the screen". Joumal of Educational Television Vol. 16, Núm. 2, pp. 87-93. 